\title{
An Ishikawa-Hybrid Proximal Point Algorithm for Nonlinear Set-Valued Inclusions Problem Based on $(A, \eta)$-Accretive Framework
}

\author{
Hong Gang Li, ${ }^{1}$ An Jian $\mathrm{Xu}_{,}{ }^{1}$ and Mao Ming Jin ${ }^{2}$ \\ ${ }^{1}$ Institute of Applied Mathematics Research, Chongqing University of Posts and Telecommunications, \\ Chongqing 400065, China \\ 2 Institute of Nonlinear Analysis Research, Changjiang Normal University, Fuling Chongqing, \\ 400803, China \\ Correspondence should be addressed to Hong Gang Li, lihg12@126.com
}

Received 30 April 2010; Accepted 8 June 2010

Academic Editor: Massimo Furi

Copyright (C) 2010 Hong Gang Li et al. This is an open access article distributed under the Creative Commons Attribution License, which permits unrestricted use, distribution, and reproduction in any medium, provided the original work is properly cited.

\begin{abstract}
A general nonlinear framework for an Ishikawa-hybrid proximal point algorithm using the notion of $(A, \eta)$-accretive is developed. Convergence analysis for the algorithm of solving a nonlinear set-valued inclusions problem and existence analysis of solution for the nonlinear set-valued inclusions problem are explored along with some results on the resolvent operator corresponding to $(A, \eta)$-accretive mapping due to Lan-Cho-Verma in Banach space. The result that sequence $\left\{x_{n}\right\}$ generated by the algorithm converges linearly to a solution of the nonlinear set-valued inclusions problem with the convergence rate $\theta$ is proved.
\end{abstract}

\section{Introduction}

The set-valued inclusions problem, which was introduced and studied by Di Bella [1], Huang et al. [2], and Jeong [3], is a useful extension of the mathematics analysis. And the variational inclusion(inequality) is an important context in the set-valued inclusions problem. It provides us with a unified, natural, novel, innovative, and general technique to study a wide class of problems arising in different branches of mathematical and engineering sciences. Various variational inclusions have been intensively studied in recent years. Ding and Luo [4], Verma [5], Huang [6], Fang and Huang [7], Lan et al. [8], Fang et al. [9], and Zhang et al. [10] introduced the concepts of $\eta$-subdifferential operators, maximal $\eta$-monotone operators, $H$-monotone operators, $A$-monotone operators, $(H, \eta)$ monotone operators, $(A, \eta)$-accretive mappings, $(G, \eta)$-monotone operators, and defined 
resolvent operators associated with them, respectively. Moreover, by using the resolvent operator technique, many authors constructed some approximation algorithms for some nonlinear variational inclusions in Hilbert spaces or Banach spaces. Recently, Verma has developed a hybrid version of the Eckstein and Bertsekas [11] proximal point algorithm, introduced the algorithm based on the $(A, \eta)$-maximal monotonicity framework [12], and studied convergence of the algorithm.

On the other hand, in 2008, $\mathrm{Li} \mathrm{[13]} \mathrm{studied} \mathrm{the} \mathrm{existence} \mathrm{of} \mathrm{solutions} \mathrm{and} \mathrm{the} \mathrm{stability}$ of perturbed Ishikawa iterative algorithm for nonlinear mixed quasivariational inclusions involving $(\mathrm{A}, \eta)$-accretive mappings in Banach spaces by using the resolvent operator technique in [14].

Inspired and motivated by recent research work in this field, in this paper, a general nonlinear framework for a Ishikawa-hybrid proximal point algorithm using the notion of $(A, \eta)$-accretive is developed. Convergence analysis for the algorithm of solving a nonlinear set-valued inclusions problem and existence analysis of solution for the nonlinear setvalued inclusions problem are explored along with some results on the resolvent operator corresponding to $(A, \eta)$-accretive mapping due to Lan et al. in Banach space. The result that sequence $\left\{x_{n}\right\}$ generated by the algorithm converges linearly to a solution of the nonlinear set-valued inclusions problem as the convergence rate $\theta$ is proved.

\section{Preliminaries}

Let $X$ be a real Banach space with dual space $X^{*}$ and $\langle\cdot, \cdot\rangle$ and let the dual pair between $X$

and $X^{*}, 2^{X}$ denote the family of all the nonempty subsets of $X$ and $\operatorname{CB}(X)$ the family of all nonempty closed bounded subsets of $X$. The generalized duality mapping $J_{q}: X \rightarrow 2^{X^{*}}$ is defined by

$$
J_{q}(x)=\left\{f^{*} \in X^{*}:\left\langle x, f^{*}\right\rangle=\|x\|^{q},\left\|f^{*}\right\|=\|x\|^{q-1}\right\}, \quad \forall x \in X
$$

where $q>1$ is a constant.

The modulus of smoothness of $X$ is the function $\rho_{X}:[0, \infty) \rightarrow[0, \infty)$ defined by

$$
\rho_{X}(t)=\sup \left\{\frac{1}{2}(\|x+y\|+\|x-y\|)-1:\|x\| \leq 1, \quad\|y\| \leq t\right\}
$$

A Banach space $X$ is called uniformly smooth if

$$
\lim _{t \rightarrow 0} \frac{\rho_{X}(t)}{t}=0
$$

$X$ is called $q$-uniformly smooth if there exists a constant $c>0$ such that

$$
\rho_{X}(t) \leq c t^{q}, \quad(q>1) .
$$


Remark 2.1. In particular, $J_{2}$ is the usual normalized duality mapping, and $J_{q}(x)=\|x\|^{q-2} J_{2}(x)$ (for all $x \neq 0$ ). If $X^{*}$ is strictly convex [15], or $X$ is uniformly smooth Banach space, then $J_{q}$ is single valued. In what follows we always denote the single-valued generalized duality mapping by $J_{q}$ in real uniformly smooth Banach space $X$ unless otherwise stated.

Let $A, Q, g: X \rightarrow X ; \eta, N: X \times X \rightarrow X$ be single-valued mappings. Let $M: X \times X \rightarrow$ $2^{X}$ be a set-valued $(A, \eta)$-accretive mapping. We consider nonlinear set-valued mixed variational inclusions problem with $(A, \eta)$-accretive mappings (NSVMVIP).

For any $u \in X$, finding $x \in X, y=Q(x)$ such that

$$
u \in N(y, g(x))+M(y) .
$$

Remark 2.2. A special case of problem (2.5) is the following.

(i) If $X=X^{*}$ is a Hilbert space, $N=0$ is the zero operator in $X, Q=I$ is the identity operator in $X$, and $u=0$, then problem (2.5) becomes the parametric usual variational inclusion $0 \in M(\mathrm{x})$ with a $(A, \eta)$-maximal monotone mapping $M$, which was studied by Verma [12].

(ii) If $X$ is a real Banach space, $Q=I$ is the identity operator in $X$, and $u=0$, then problem (2.5) becomes the parametric usual variational inclusion $u \in N(x, g(x))+$ $M(x)$ with a $(A, \eta)$-accretive mapping, which was studied by Li [13].

It is easy to see that a number of known special classes of variational inclusions and variational inequalities in the problem (2.5) are studied (see [2, 7, 12-14]).

Let us recall the following results and concepts.

Definition 2.3. A single-valued mapping $\eta: X \times X \rightarrow X$ is said to be $\tau$-Lipschitz continuous if there exists a constant $\tau>0$ such that

$$
\|\eta(x, y)\| \leq \tau\|x-y\|, \quad \forall x, y \in X
$$

Definition 2.4. A single-valued mapping $A: X \rightarrow X$ is said to be

(i) accretive if

$$
\left\langle A\left(x_{1}\right)-A\left(x_{2}\right), J_{q}\left(x_{1}-x_{2}\right)\right\rangle \geq 0, \quad \forall x_{1}, x_{2} \in X,
$$

(ii) strictly accretive, if $A$ is accretive and $\left\langle A\left(x_{1}\right)-A\left(x_{2}\right), J_{q}\left(x_{1}-x_{2}\right)\right\rangle=0$ if and only if $x_{1}=x_{2}$ for all $x_{1}, x_{2} \in X$,

(iii) $r$-strongly $\eta$-accretive if there exists a constant $r>0$ such that

$$
\left\langle A\left(x_{1}\right)-A\left(x_{2}\right), J_{q}\left(\eta\left(x_{1}, x_{2}\right)\right)\right\rangle \geq r\left\|x_{1}-x_{2}\right\|^{q}, \quad \forall x_{1}, x_{2} \in X,
$$

(iv) $\alpha$-Lipschitz continuous if there exists a constant $\alpha>0$ such that

$$
\left\|A\left(x_{1}\right)-A\left(x_{2}\right)\right\| \leq \alpha\left\|x_{1}-x_{2}\right\|, \quad \forall x_{1}, x_{2} \in X .
$$


Definition 2.5. A single-valued mapping $N: X \times X \rightarrow X$ is said to be

(i) $(\mu, v)$-Lipschitz continuous if there exist constants $\mu, v>0$ such that

$$
\left\|N\left(x_{1}, y_{1}\right)-N\left(x_{2}, y_{2}\right)\right\| \leq \mu\left\|\mathrm{x}_{1}-x_{2}\right\|+v\left\|y_{1}-y_{2}\right\| \quad \forall x_{i}, y_{i} \in X, \quad i=1,2
$$

(ii) $(\psi, \kappa)$-Q-relaxed cocoercive with respect to $A Q$ in the first argument if there exist constants $\psi, \kappa>0$, and for all $x_{i} \in X, y_{i}=Q\left(x_{i}\right)(i=1,2)$ such that

$$
\left\langle N\left(y_{1}, \cdot\right)-N\left(y_{2}, \cdot\right), J_{q}\left(A\left(y_{1}\right)-A\left(y_{2}\right)\right)\right\rangle \geq-\psi\left\|N\left(y_{1}, \cdot\right)-N\left(y_{2}, \cdot\right)\right\|^{q}+\kappa\left\|x_{1}-x_{2}\right\|^{q},
$$

where $A, Q: X \rightarrow X$ are single-valued mappings.

Definition 2.6. Let $A: X \rightarrow X$, and let $\eta: X \times X \rightarrow X$ be single-valued mappings. A setvalued mapping $M: X \rightarrow 2^{X}$ is said to be

(i) accretive if

$$
\left\langle u_{1}-u_{2}, J_{q}\left(x_{1}-x_{2}\right)\right\rangle \geq 0, \quad \forall x_{1}, x_{2} \in X, \quad u_{1} \in M\left(x_{1}\right), \quad u_{2} \in M\left(x_{2}\right) ;
$$

(ii) $\eta$-accretive if

$$
\left\langle u_{1}-u_{2}, J_{q}\left(\eta\left(x_{1}, x_{2}\right)\right)\right\rangle \geq 0, \quad \forall x_{1}, x_{2} \in X, \quad u_{1} \in M\left(x_{1}\right), \quad u_{2} \in M\left(x_{2}\right)
$$

(iii) $r$-strongly accretive if there exists a constant $r>0$ such that

$$
\left\langle y_{1}-y_{2}, J_{q}\left(x_{1}-x_{2}\right)\right\rangle \geq r\left\|x_{1}-x_{2}\right\|^{q}, \quad \forall x_{i} \in X, \quad y_{i} \in M\left(x_{i}\right) \quad(i=1,2)
$$

(iv) $m$-relaxed $\eta$-accretive if there exists a constant $m>0$ such that

$$
\left\langle u_{1}-u_{2}, J_{q}\left(\eta\left(x_{1}, x_{2}\right)\right)\right\rangle \geq-m\left\|x_{1}-x_{2}\right\|^{q}, \quad \forall x_{1}, x_{2} \in X, \quad u_{1} \in M\left(x_{1}\right), \quad u_{2} \in M\left(x_{2}\right),
$$

(v) $A$-accretive, if $M$ is accretive and $(A+\rho M)(X)=X$ for all $\rho>0$,

(vi) $(A, \eta)$-accretive if $M$ is $m$-relaxed $\eta$-accretive and $(A+\rho M)(X)=X$ for all $\rho>0$. 
Based on the literature [8], we can define the resolvent operator $R_{\rho, M}^{A, \eta}$ as follows.

Definition 2.7 (see [8]). Let $\eta: X \times X \rightarrow X$ be a single-valued mapping, $A: X \rightarrow X$ a strictly $\eta$-accretive single-valued mapping and $M: X \times X \rightarrow 2^{X}$ a $(A, \eta)$-accretive mapping. The resolvent operator $R_{\rho, M}^{A, \eta}: X \rightarrow X$ is defined by

$$
R_{\rho, M}^{A, \eta}(x)=(A+\rho M)^{-1}(x), \quad \forall x \in X
$$

where $\rho>0$ is a constant.

Remark 2.8. The $(A, \eta)$-accretive mappings are more general than $(H, \eta)$-monotone mappings and $m$-accretive mappings in Banach space or Hilbert space, and the resolvent operators associated with $(A, \eta)$-accretive mappings include as special cases the corresponding resolvent operators associated with $(H, \eta)$-monotone operators, $m$-accretive mappings, $A$ monotone operators, $\eta$-subdifferential operators $[3-14,16,17]$.

Lemma 2.9 (see [8]). Let $\eta: X \times X \rightarrow X$ be $\tau$-Lipschtiz continuous mapping, $A: X \rightarrow X$ be an $r$-strongly $\eta$-accretive mapping, and $M: X \times X \rightarrow 2^{X}$ an $(A, \eta)$-accretive mapping. Then the generalized resolvent operator $R_{\rho, M}^{A, \eta}: X \rightarrow X$ is $\tau^{q-1} /(r-m \rho)$-Lipschitz continuous, that is,

$$
\left\|R_{\rho, M}^{A, \eta}(x)-R_{\rho, M}^{A, \eta}(y)\right\| \leq \frac{\tau^{q-1}}{r-m \rho}\|x-y\|, \quad \forall x, y \in X,
$$

where $\rho \in(0, r / m)$.

In the study of characteristic inequalities in q-uniformly smooth Banach spaces, $\mathrm{Xu}$ [18] proved the following result.

Lemma 2.10 (see [18]). Let $X$ be a real uniformly smooth Banach space. Then $X$ is q-uniformly smooth if and only if there exists a constant $c_{q}>0$ such that for all $x, y \in X$,

$$
\|x+y\|^{q} \leq\|x\|^{q}+q\left\langle y, J_{q}(x)\right\rangle+c_{q}\|y\|^{q} .
$$

\section{The Existence of Solutions}

Now, we are studing the existence for solutions of problem (2.5).

Lemma 3.1. Let $X$ be a Banach space. Let $\eta: X \times X \rightarrow X$ be a $\tau$-Lipschtiz continuous mapping, $A: X \rightarrow X$ be an r-strongly $\eta$-accretive mapping, and $M: X \rightarrow 2^{X}$ an $(A, \eta)$-accretive mapping. Then the following statements are mutually equivalent.

(i) An element $x \in X$ is a solution of problem (2.5).

(ii) For a $x \in X$ and any $1>\lambda>0$, there exists $y=Q(x)$ such that

$$
x=(1-\lambda) x+\lambda\left(x-y+R_{\rho, M}^{A, \eta}(A(y)-\rho N(y, g(x))+\rho u)\right),
$$

where $\rho>0$ is a constant.

Proof. This directly follows from the definition of $R_{\rho, M(x)}^{A, \eta}$. 
Theorem 3.2. Let $X$ be a q-uniformly smooth Banach space. Let $A, Q, g: X \rightarrow X ; \eta, N: X \times$ $X \rightarrow X$ be single-valued mappings, and $\eta$ be a $\tau$-Lipschtiz continuous mapping, $A$ a $r$-strongly $\eta$ accretive and $\alpha$-Lipschitz continuous mapping, $Q$ be a $\gamma$-strongly accretive and $X$-Lipschitz continuous mapping, and $g$ a $\varphi$-Lipschitz continuous mapping, respectively. Let $N: X \times X \rightarrow X$ be $(\mu, v)$ Lipschitz continuous, and $(\psi, \kappa)$-Q-relaxed cocoercive with respect to $A Q$ in the first argument. Let $M: X \rightarrow 2^{X}$ be a set-valued $(A, \eta)$-accretive mapping. If the following condition holds:

$$
\tau^{q}\left[\left(\alpha^{q}+c_{q} \rho^{q} \mu^{q} x^{q}+q \rho \psi \mu^{q} x^{q}-q \rho \kappa\right)^{1 / q}+\rho v \varphi\right]<\tau(r-m \rho)\left(1-\left(1+c_{q} x^{q}-q \gamma\right)^{1 / q}\right)
$$

where $c_{q}>0$ is the same as in Lemma 2.10, and $\rho \in(0, r / m)$, then the problem (2.5) has a solution $x^{*} \in X$.

Proof. Define a mapping $F: X \rightarrow X$ as follows:

$$
F(x)=(1-\lambda) x+\lambda\left(x-y+R_{\rho, M}^{A, \eta}(A(y)-\rho N(y, g(x))+\rho u)\right), \quad \forall x \in X
$$

For elements $x_{1}, x_{2} \in X$, if we let $y_{i}=Q\left(x_{i}\right)$ and

$$
s_{i}=A\left(y_{i}\right)-\rho N\left(y_{i}, g\left(x_{i}\right)\right)+\rho u \quad(i=1,2),
$$

then by (3.1), (3.3), and Lemma 2.10, we have

$$
\begin{aligned}
\| F\left(x_{1}\right) & -F\left(x_{2}\right)\|=\|(1-\lambda) x_{1}+\lambda\left(x_{1}-y_{1}+R_{\rho, M}^{A, \eta}\left(s_{1}\right)\right)-(1-\lambda) x_{2}-\lambda\left(x_{2}-y_{2}+R_{\rho, M}^{A, \eta}\left(s_{2}\right)\right) \| \\
\leq & (1-\lambda)\left\|x_{1}-x_{2}\right\|+\lambda\left\|x_{1}-x_{2}-\left(y_{1}-y_{2}\right)\right\|+\lambda\left\|R_{\rho, M}^{A, \eta}\left(s_{2}\right)-R_{\rho, M}^{A, \eta}\left(s_{2}\right)\right\| \\
\leq & (1-\lambda)\left\|x_{1}-x_{2}\right\|+\lambda \frac{\tau^{q-1}}{r-m \rho}\left(\rho\left\|N\left(y_{2}, g\left(x_{1}\right)\right)-N\left(y_{2}, g\left(x_{2}\right)\right)\right\|\right. \\
& \left.+\left\|A\left(y_{1}\right)-A\left(y_{2}\right)-\rho\left(N\left(y_{1}, g\left(x_{1}\right)\right)-N\left(y_{2}, g\left(x_{1}\right)\right)\right)\right\|\right)+\lambda\left\|x_{1}-x_{2}-\left(y_{1}-y_{2}\right)\right\| .
\end{aligned}
$$

Since $N(\cdot, \cdot)$ is $(\psi, \kappa)$-Q-relaxed cocoercive with respect to $A Q$ in the first argument and $Q$ is a $X$-Lipschitz continuous mapping so we obtain

$$
\begin{aligned}
& \left\|A\left(y_{1}\right)-A\left(y_{2}\right)-\rho\left(N\left(y_{1}, g\left(x_{1}\right)\right)-N\left(y_{2}, g\left(x_{1}\right)\right)\right)\right\|^{q} \\
& \leq\left\|A\left(y_{1}\right)-A\left(y_{2}\right)\right\|^{q}+c_{q} \rho^{q}\left\|N\left(y_{1}, g\left(x_{1}\right)\right)-N\left(y_{2}, g\left(x_{1}\right)\right)\right\|^{q} \\
& -q \rho\left\langle N\left(y_{1}, \cdot\right)-N\left(y_{2}, \cdot\right), J_{q}\left(A\left(y_{1}\right)-A\left(y_{2}\right)\right)\right\rangle \\
& \leq\left[\alpha^{q} x^{q}+c_{q} \rho^{q} \mu^{q} x^{q}+q \rho \psi \mu^{q} x^{q}-q \rho \kappa\right]\left\|x_{1}-x_{2}\right\|^{q}, \\
& \left\|N\left(y_{2}, g\left(x_{1}\right)\right)-N\left(y_{2}, g\left(x_{2}\right)\right)\right\| \leq v \varphi\left\|x_{1}-x_{2}\right\| \text {. }
\end{aligned}
$$


Fixed Point Theory and Applications

By $\gamma$-strongly accretivity of $Q$, we have

$$
\begin{aligned}
\| x_{1}- & x_{2}-\left(y_{1}-y_{2}\right) \|^{q} \\
\leq & \left\|x_{1}-x_{2}\right\|^{q}+c_{q}\left\|y_{1}-y_{2}\right\|^{q} \\
& -q\left\langle y_{1}-y_{2}, J_{q}\left(x_{1}-x_{2}\right)\right\rangle \\
\leq & \left(1+c_{q} x^{q}-q \gamma\right)\left\|x_{1}-x_{2}\right\|^{q} .
\end{aligned}
$$

Combining(3.5), (3.6), (3.7), and (3.8), we can get

$$
\left\|F\left(x_{1}\right)-F\left(x_{2}\right)\right\| \leq[(1-\lambda)+\lambda \theta]\left\|x_{1}-x_{2}\right\|,
$$

where

$$
\theta=\left(1+c_{q} X^{q}-q \gamma\right)^{1 / q}+\frac{\tau^{q-1}}{r-m \rho}\left[\left(\alpha^{q}+c_{q} \rho^{q} \mu^{q} X^{q}+q \rho \psi \mu^{q} X^{q}-q \rho \kappa\right)^{1 / q}+\rho v \varphi\right]
$$

It follows from (3.2) and (3.9) that $F$ has a fixed point in $X$, that is, there exists a point $x^{*} \in X$ such that $x^{*} \in \widetilde{G}\left(x^{*}\right)$, and

$$
x^{*}=(1-\lambda) x^{*}+\lambda\left(x^{*}-y^{*}+R_{\rho, M}^{A, \eta}\left(A\left(y^{*}\right)-\rho N\left(y^{*}, g\left(x^{*}\right)\right)+\rho u\right)\right)
$$

where $y^{*}=Q\left(x^{*}\right)$. This completes the proof.

\section{Ishikawa-Hybrid Proximal Point Algorithm}

Based on Lemma 3.1, we develop an Ishikawa-hybrid proximal point algorithm for finding an iterative sequence solving problem (2.5) as follows.

Algorithm 4.1. Let $x^{*}$ be a solution of problem (2.5). Let $\left\{\alpha_{n}\right\}_{n=0}^{\infty},\left\{\beta_{n}\right\}_{n=0}^{\infty},\left\{a_{n}\right\}_{n=0}^{\infty},\left\{b_{n}\right\}_{n=0}^{\infty}$ and $\left\{\rho_{n}\right\}_{n=0}^{\infty}$, be five nonnegative sequences such that

$$
\begin{array}{r}
\lim _{n \rightarrow \infty} a_{n}=\lim _{n \rightarrow \infty} b_{n}=0, \quad a=\limsup _{n \rightarrow \infty} \alpha_{n}<1, \quad b=\limsup _{n \rightarrow \infty} \beta_{n}<1, \quad \rho_{n} \uparrow \rho \leq \infty, \\
(n=0,1,2, \ldots) .
\end{array}
$$


Step 1. For an arbitrarily initial point $x_{0} \in X$, we choose suitable $z_{0} \in X$, letting

$$
\begin{gathered}
y_{0}=\left(1-\beta_{0}\right) x_{0}+\beta_{0} z_{0}, \\
\left\|z_{0}-R_{\rho_{0}, M}^{A, \eta}\left(A\left(Q\left(x_{0}\right)\right)-\rho_{0} N\left(Q\left(x_{0}\right), g\left(x_{0}\right)\right)+\rho_{0} u\right)\right\| \leq b_{0}\left\|z_{0}-x_{0}\right\|, \\
x_{1}=\left(1-\alpha_{0}\right) x_{0}+\alpha_{0} w_{0}, \\
\left\|w_{0}-R_{\rho_{0}, M}^{A, \eta}\left(A\left(Q\left(y_{0}\right)\right)-\rho_{0} N\left(Q\left(y_{0}\right), g\left(x_{0}\right)\right)+\rho_{0} u\right)\right\| \leq a_{0}\left\|w_{0}-y_{0}\right\| .
\end{gathered}
$$

Step 2. The sequences $\left\{x_{n}\right\}$ and $\left\{y_{n}\right\}$ are generated by an iterative procedure

$$
\begin{gathered}
y_{n}=\left(1-\beta_{n}\right) x_{n}+\beta_{n} z_{n}, \\
\left\|z_{n}-R_{\rho_{n}, M}^{A, \eta}\left(A\left(Q\left(x_{n}\right)\right)-\rho_{n} N\left(Q\left(x_{n}\right), g\left(x_{n}\right)\right)+\rho_{n} u\right)\right\| \leq b_{n}\left\|z_{n}-x_{n}\right\|, \\
x_{n+1}=\left(1-\alpha_{n}\right) x_{n}+\alpha_{n} w_{n}, \\
\left\|w_{n}-R_{\rho_{n}, M}^{A, \eta}\left(A\left(Q\left(y_{n}\right)\right)-\rho_{n} N\left(Q\left(y_{n}\right), g\left(x_{n}\right)\right)+\rho_{n} u\right)\right\| \leq a_{n}\left\|w_{n}-y_{n}\right\|,
\end{gathered}
$$

where $n=1,2, \ldots$

Remark 4.2. For a suitable choice of the mappings $A, \eta, Q, N, g, M$, space $X$, and nonnegative sequences $\left\{a_{n}\right\}_{n=0}^{\infty},\left\{b_{n}\right\}_{n=0}^{\infty}$, Algorithm 4.1 can be degenerated to a number of algorithms involving many known algorithms which are due to classes of variational inequalities and variational inclusions [12-14].

Theorem 4.3. Let $X, A, N, Q, g$, and $M$ be the same as in Theorem 3.2, then condition (3.2) holds. Let $\left\{\alpha_{n}\right\}_{n=0}^{\infty},\left\{\beta_{n}\right\}_{n=0}^{\infty},\left\{a_{n}\right\}_{n=0}^{\infty},\left\{b_{n}\right\}_{n=0}^{\infty}$ and $\left\{\rho_{n}\right\}_{n=0}^{\infty}$ be the same as in Algorithm 4.1. Then the sequence $\left\{x_{n}\right\}$ generated by hybrid proximal point Algorithm 4.1 converges linearly to a solution $x^{*}$ of problem (2.5) as

$$
\tau^{q}\left[\left(\alpha^{q}+c_{q} \rho^{q} \mu^{q} X^{q}+q \rho \psi \mu^{q} X^{q}-q \rho \kappa\right)^{1 / q}+\rho v \varphi\right]<\tau(r-m \rho)\left(1-\left(1+c_{q} X^{q}-q \gamma\right)^{1 / q}\right),
$$

where $c_{q}>0$ is the same as in Lemma 2.10, $\rho \in(0, r / m)$, and the convergence rate is

$$
\theta=(1-a)+a\left[\frac{\tau^{q-1}}{r-m \rho}\left(\sqrt[q]{\alpha^{q}+q c_{q} \psi \mu^{q} \rho-q \kappa \rho+\mu^{q} \rho^{q}}+\rho v \varphi\right)+\left(1+c_{q} \chi^{q}-q \gamma\right)^{1 / q}\right]
$$

Proof. Suppose that the sequence $\left\{x_{n}\right\}$ is the the sequence generated by the Ishikawa-hybrid proximal point Algorithm 4.1, and that $x^{*}$ is a solution of problem (2.5). From Lemma 3.1 and condition $\alpha_{n} \in[0,1)$, we can get

$$
x^{*}=\left(1-\alpha_{n}\right) x^{*}+\alpha_{n}\left(x^{*}-y^{*}+R_{\rho_{n}, M}^{A, \eta}\left(A\left(y^{*}\right)-\rho_{n} N\left(y^{*}, g\left(x^{*}\right)\right)+\rho_{n} u\right)\right),
$$

where $y^{*}=Q\left(x^{*}\right)$. 
Fixed Point Theory and Applications

For all $n \geq 0$, and $y_{n}=Q\left(x_{n}\right)$, setting

$$
u_{n+1}=\left(1-\alpha_{n}\right) x_{n}+\alpha_{n}\left(x_{n}-y_{n}+R_{\rho_{n}, M}^{A, \eta}\left(A\left(y_{n}\right)-\rho_{n} N\left(y_{n}, g\left(x_{n}\right)\right)+\rho_{n} u\right)\right),
$$

we find the estimation

$$
\begin{aligned}
& \left\|u_{n+1}-x^{*}\right\| \leq\left(1-\alpha_{n}\right)\left\|x_{n}-x^{*}\right\|+\alpha_{n} \\
& \times \| R_{\rho_{n}, M}^{A, \eta}\left(A\left(y_{n}\right)-\rho_{n} N\left(y_{n}, g\left(x_{n}\right)\right)+\rho_{n} u\right) \\
& -R_{\rho_{n}, M}^{A, \eta}\left(A\left(y^{*}\right)-\rho_{n} N\left(y^{*}, g\left(x^{*}\right)\right)+\rho_{n} u\right) \| \\
& +\alpha_{n}\left\|x^{*}-x_{n}-\left(y^{*}-y_{n}\right)\right\| \leq\left(1-\alpha_{n}\right)\left\|x_{n}-x^{*}\right\|+\alpha_{n} \frac{\tau^{q-1}}{r-m \rho_{n}} \\
& \times\left[\left\|A\left(y_{n}\right)-A\left(y^{*}\right)-\rho_{n}\left(N\left(y_{n}, g\left(x_{n}\right)\right)-N\left(y^{*}, g\left(x_{n}\right)\right)\right)\right\|\right. \\
& \left.\quad+\rho_{n}\left\|N\left(y^{*}, g\left(x_{n}\right)\right)-N\left(y^{*}, g\left(x^{*}\right)\right)\right\|\right]+\alpha_{n}\left\|x^{*}-x_{n}-\left(y^{*}-y_{n}\right)\right\| .
\end{aligned}
$$

By the conditions and Lemma 2.10, we have

$$
\begin{aligned}
& \left\|A\left(y_{n}\right)-A\left(y^{*}\right)-\rho_{n}\left(N\left(y_{n}, g\left(x_{n}\right)\right)-N\left(y^{*}, g\left(x_{n}\right)\right)\right)\right\|^{q} \\
& \leq\left\|A\left(y_{n}\right)-A\left(y^{*}\right)\right\|^{q}+c_{q} \rho_{n}^{q}\left\|N\left(y_{n}, g\left(x_{n}\right)\right)-N\left(y^{*}, g\left(x_{n}\right)\right)\right\|^{q} \\
& -q \rho_{n}\left\langle N\left(y_{n}, g\left(x_{n}\right)\right)-N\left(y^{*}, g\left(x_{n}\right)\right), J_{q}\left(A\left(y_{n}\right)-A\left(y^{*}\right)\right)\right\rangle \\
& \leq\left[\alpha^{q} x^{q}+c_{q} \rho_{n}^{q} \mu^{q} x^{q}+q \rho_{n} \psi \mu^{q} x^{q}-q \rho_{n} \kappa\right]\left\|x_{n}-x^{*}\right\|^{q}, \\
& \left\|N\left(x^{*}, g\left(x_{n}\right)\right)-N\left(x^{*}, g\left(x^{*}\right)\right)\right\| \leq v \varphi\left\|x_{n}-x^{*}\right\|, \\
& \left\|x^{*}-x_{n}-\left(y^{*}-y_{n}\right)\right\| \leq\left(1+c_{q} x^{q}-q r\right)\left\|x^{*}-x_{n}\right\|^{q} \text {. }
\end{aligned}
$$

It follows from (4.8)-(4.10) that

$$
\left\|u_{n+1}-x^{*}\right\| \leq \theta_{n}\left\|x_{n}-x^{*}\right\|
$$

where

$$
\begin{gathered}
\theta_{n}=\left(1-\alpha_{n}\right)+\alpha_{n} h_{n} \\
h_{n}=\frac{\tau^{q-1}}{r-m \rho_{n}}\left(\sqrt[q]{\alpha^{q} X^{q}+c_{q} \rho_{n}^{q} \mu^{q} X^{q}+q \rho_{n} \psi \mu^{q} X^{q}-q \rho_{n} \kappa}+\rho_{n} v \varphi\right)+\left(1+c_{q} X^{q}-q \gamma\right)^{1 / q} .
\end{gathered}
$$


Since $x_{n+1}=\left(1-\alpha_{n}\right) x_{n}+\alpha_{n} w_{n}$ and (4.3), $x_{n+1}-x_{n}=\alpha_{n}\left(w_{n}-x_{n}\right)$ and

$$
\begin{aligned}
\left\|x_{n+1}-u_{n+1}\right\| \leq & \|\left(1-\alpha_{n}\right) x_{n}+\alpha_{n} w_{n} \\
& -\left[\left(1-\alpha_{n}\right) x_{n}+\alpha_{n}\left(x_{n}-y_{n}+R_{\rho_{n}, M}^{A, \eta}\left(A\left(y_{n}\right)-\rho_{n} N\left(y_{n}, g\left(x_{n}\right)\right)+\rho_{n} u\right)\right)\right] \| \\
& \leq \alpha_{n}\left\|w_{n}-R_{\rho_{n}, M}^{A, \eta}\left(A\left(y_{n}\right)-\rho_{n} N\left(y_{n}, g\left(x_{n}\right)\right)+\rho_{n} u\right)\right\|+\alpha_{n}\left\|x_{n}-y_{n}\right\| \\
& \leq \alpha_{n} a_{n}\left\|w_{n}-y_{n}\right\|+\alpha_{n}\left\|x_{n}-y_{n}\right\| .
\end{aligned}
$$

Next, we calculate

$$
\begin{aligned}
\left\|x_{n+1}-x^{*}\right\| & \leq\left\|u_{n+1}-x^{*}\right\|+\left\|x_{n+1}-u_{n+1}\right\| \leq\left\|u_{n+1}-x^{*}\right\|+\alpha_{n} a_{n}\left\|w_{n}-y_{n}\right\|+\alpha_{n}\left\|x_{n}-y_{n}\right\| \\
& \leq\left\|u_{n+1}-x^{*}\right\|+\alpha_{n} a_{n}\left(\left\|w_{n}-x_{n}\right\|+\left\|y_{n}-x_{n}\right\|\right)+\alpha_{n}\left\|x_{n}-y_{n}\right\| \\
& \leq\left\|u_{n+1}-x^{*}\right\|+\alpha_{n} a_{n}\left\|w_{n}-x_{n}\right\|+\alpha_{n}\left(a_{n}+1\right)\left\|y_{n}-x_{n}\right\| \\
& \leq \theta_{n}\left\|x_{n}-x^{*}\right\|+a_{n}\left\|x^{*}-x_{n}\right\|+a_{n}\left\|x_{n+1}-x^{*}\right\|+\alpha_{n}\left(a_{n}+1\right)\left(\left\|y_{n}-x^{*}\right\|+\left\|x^{*}-x_{n}\right\|\right) \\
& \leq \theta_{n}\left\|x_{n}-x^{*}\right\|+a_{n}\left\|x_{n+1}-x^{*}\right\|+\alpha_{n}\left(a_{n}+1\right)\left\|y_{n}-x^{*}\right\|+\left(a_{n}+\alpha_{n}\left(a_{n}+1\right)\right)\left\|x^{*}-x_{n}\right\| .
\end{aligned}
$$

This implies that

$$
\left\|x_{n+1}-x^{*}\right\| \leq \frac{\theta_{n}+a_{n}+\left(1+\alpha_{n}\right) a_{n}}{1-a_{n}}\left\|x_{n}-x^{*}\right\|+\frac{\left(1+\alpha_{n}\right) a_{n}}{1-a_{n}}\left\|y_{n}-x^{*}\right\|,
$$

letting

$$
x^{*}=\left(1-\beta_{n}\right) x^{*}+\beta_{n}\left(x^{*}-y^{*}+R_{\rho_{n}, M}^{A, \eta}\left(A\left(y^{*}\right)-\rho_{n} N\left(y^{*}, g\left(x^{*}\right)\right)+\rho_{n} u\right)\right) .
$$

For all $n \geq 0$, set

$$
v_{n}=\left(1-\beta_{n}\right) x_{n}+\beta_{n}\left(x_{n}-y_{n}+R_{\rho_{n}, M}^{A, \eta}\left(A\left(y_{n}\right)-\rho_{n} N\left(y_{n}, g\left(x_{n}\right)\right)+\rho_{n} u\right)\right) .
$$

For the same reason,

$$
\left\|v_{n}-x^{*}\right\| \leq \vartheta_{n}\left\|x_{n}-x^{*}\right\|
$$

where

$$
\vartheta_{n}=\left(1-\beta_{n}\right)+\beta_{n} h_{n}
$$


Fixed Point Theory and Applications

$$
\left\|y_{n}-x^{*}\right\| \leq \vartheta_{n}\left\|x_{n}-x^{*}\right\|+\left(b_{n}+b_{n} \beta_{n}+\beta_{n}\right)\left(\left\|x^{*}-x_{n}\right\|+\left\|x^{*}-y_{n}\right\|\right) .
$$

Furthermore,

$$
\left\|y_{n}-x^{*}\right\| \leq \frac{\left(\vartheta_{n}+b_{n}+b_{n} \beta_{n}+\beta_{n}\right)}{1-\left(b_{n}+b_{n} \beta_{n}+\beta_{n}\right)}\left\|x_{n}-x^{*}\right\|
$$

Combining (4.16)-(4.22), then we have

$$
\begin{aligned}
\left\|x_{n+1}-x^{*}\right\| \leq & {\left[\frac{\theta_{n}+a_{n}+\left(1+\alpha_{n}\right) a_{n}}{1-a_{n}}\right.} \\
& \left.+\frac{\left(1+\alpha_{n}\right) a_{n}}{1-a_{n}} \frac{\left(\vartheta_{n}+b_{n}+b_{n} \beta_{n}+\beta_{n}\right)}{1-\left(b_{n}+b_{n} \beta_{n}+\beta_{n}\right)}\right]\left\|x_{n}-x^{*}\right\| .
\end{aligned}
$$

By (4.4) and the condition $\lim _{n \rightarrow \infty} a_{n}=\lim _{n \rightarrow \infty} b_{n}=0$, we can see that

$$
\begin{aligned}
\theta=\vartheta=\limsup _{n \rightarrow \infty} & \theta_{n} \\
=(1-a)+a & {\left[\frac{\tau^{q-1}}{r-m \rho}\left(\sqrt[q]{\alpha^{q}+q c_{q} \psi \mu^{q} \rho-q \kappa \rho+\mu^{q} \rho^{q}}+\rho v \varphi\right)\right.} \\
& \left.+\left(1+c_{q} x^{q}-q \gamma\right)^{1 / q}\right]<1
\end{aligned}
$$

and the convergence rate is $\theta$.By (4.4), if $h=\lim _{n \rightarrow \infty} h_{n}$, then it follows that $0<h<1$ and $0<\theta<1$. Therefor, the sequence $\left\{x_{n}\right\}$ generated hybrid proximal point Algorithm 4.1 converges linearly to a solution $x^{*}$ of problem (2.5) with convergence rate $\theta$. This completes the proof.

Remark 4.4. For a suitable choice of the mappings $A, \eta, Q, g, N$, and $M$, we can obtain several known results $[12-14,17]$ as special cases of Theorem 3.2 and Theorem 4.3.

\section{References}

[1] B. Di Bella, "An existence theorem for a class of inclusions," Applied Mathematics Letters, vol. 13, no. 3, pp. 15-19, 2000.

[2] N. J. Huang, Y. Y. Tang, and Y. P. Liu, "Some new existence theorems for nonlinear inclusion with an application," Nonlinear Functional Analysis and Applications, vol. 6, no. 3, pp. 341-350, 2001.

[3] J. U. Jeong, "Generalized set-valued variational inclusions and resolvent equations in Banach spaces," Computers \& Mathematics with Applications, vol. 47, no. 8-9, pp. 1241-1247, 2004.

[4] X. P. Ding and C. L. Luo, "Perturbed proximal point algorithms for general quasi-variational-like inclusions," Journal of Computational and Applied Mathematics, vol. 113, no. 1-2, pp. 153-165, 2000.

[5] R. U. Verma, "Approximation-solvability of a class of A-monotone variational inclusion problems," Journal KSIAM, vol. 8, no. 1, pp. 55-66, 2004.

[6] N. J. Huang, "Nonlinear implicit quasi-variational inclusions involving generalized $m$-accretive mappings," Archives of Inequalities and Applications, vol. 2, no. 4, pp. 413-425, 2004. 
[7] Y. P. Fang and N. J. Huang, "H-accretive operators and resolvent operator technique for solving variational inclusions in Banach spaces," Applied Mathematics Letters, vol. 17, no. 6, pp. 647-653, 2004.

[8] H. Y. Lan, Y. J. Cho, and R. U. Verma, "Nonlinear relaxed cocoercive variational inclusions involving $(A, \eta)$-accretive mappings in Banach spaces," Computers $\mathcal{E}$ Mathematics with Applications, vol. 51, no. 9-10, pp. 1529-1538, 2006.

[9] Y. P. Fang, N. J. Huang, and H. B. Thompson, "A new system of variational inclusions with $(H, \eta)$ -monotone operators in Hilbert spaces," Computers $\mathcal{E}$ Mathematics with Applications. An International Journal, vol. 49, no. 2-3, pp. 365-374, 2005.

[10] Q. B. Zhang, X. P. Zhanging, and C. Z. Cheng, "Resolvent operator technique for solving generalized implicit variational-like inclusions in Banach space," Journal of Mathematical Analysis and Applications, no. 20, pp. 216-221, 2007.

[11] J. Eckstein and D. P. Bertsekas, "On the Douglas-Rachford splitting method and the proximal point algorithm for maximal monotone operators," Mathematical Programming, vol. 55, no. 3, pp. 293-318, 1992.

[12] R. U. Verma, "A hybrid proximal point algorithm based on the $(A, \eta)$-maximal monotonicity framework," Applied Mathematics Letters, vol. 21, no. 2, pp. 142-147, 2008.

[13] H. G. Li, "Perturbed Ishikawa iterative algorithm and stability for nonlinear mixed quasi-variational inclusions involving $(A, \eta)$-accretive mappings," Advances in Nonlinear Variational Inequalities, vol. 11, no. 1, pp. 41-50, 2008.

[14] N. J. Huang and Y. P. Fang, "Generalized m-accretive mappings in Banach spaces," Journal of Sichuan University, vol. 38, no. 4, pp. 591-592, 2001.

[15] X. L. Weng, "Fixed point iteration for local strictly pseudo-contractive mapping," Proceedings of the American Mathematical Society, vol. 113, no. 3, pp. 727-731, 1991.

[16] S. H. Shim, S. M. Kang, N. J. Huang, and Y. J. Cho, "Perturbed iterative algorithms with errors for completely generalized strongly nonlinear implicit quasivariational inclusions," Journal of Inequalities and Applications, vol. 5, no. 4, pp. 381-395, 2000.

[17] R. P. Agarwal, Y. J. Cho, and N. J. Huang, "Sensitivity analysis for strongly nonlinear quasi-variational inclusions," Applied Mathematics Letters, vol. 13, no. 6, pp. 19-24, 2000.

[18] H. K. Xu, "Inequalities in Banach spaces with applications," Nonlinear Analysis. Theory, Methods $\mathcal{E}$ Applications, vol. 16, no. 12, pp. 1127-1138, 1991. 